\title{
Molecular Dynamics Simulation of the Effect of Carbon Nanotube Chirality on Nano-Joining with Gold Particle
}

\author{
Xiliang Qiu, Di Xu, Tiesong Lin, Xue Yang, Yu Liu and Peng He* \\ State Key Laboratory of Advanced Welding and Joining, Harbin Institute of Technology, Harbin 150001, China
}

The behavior of gold atoms depending on the CNT chirality in a nanojoining process is studied by molecular dynamics simulation. The deformation regularity and the diffusing characteristic of the gold particle during the joining process, as well as the C-Au bonds distribution in the final joint are studied. Our results show that when joining with higher spirality CNT, gold particle tends to deform more. With the CNT more similar to armchair type, the gold particle as a whole displaces more. In the final joint, the total bonds number decreases from typical armchair CNT to typical zig-zag CNT. However, the bonds distribution in detail is irregular from joint to joint, which is the consequence of lattice structure of both materials. [doi:10.2320/matertrans.MI201403]

(Received November 28, 2014; Accepted February 17, 2015; Published April 17, 2015)

Keywords: carbon nanotubes, molecular dynamics, nanojoining

\section{Introduction}

Since being found two decades ago, ${ }^{1)}$ carbon nanotubes (CNTs) have shown unique mechanical, electrical, optical and magnetic characteristics, ${ }^{2-5)}$ which have drawn intense attentions amongst the researchers worldwide. A common requirement for a CNT is its contact with metals to fulfill its applications in most fields, and one way to meet this requirement is nano-joining. ${ }^{6}$ With the help of fast developing experimental techniques such as high resolution transmission electron microscopy, scanning tunneling microscopes and atomic force microscopes, it has been successful to do experiments on a single carbon nanotube (CNT). ${ }^{7-17)}$ Taking the advantage of in situ catalyst particles in CNTs produced by catalytic chemical vapor deposition method, ${ }^{18)}$ Asaka et $a{ }^{7}{ }^{7)}$ soldered a CNT with a platinum coated needle tip. Changxin et al. ${ }^{8)}$ joined CNTs with titanium electrodes by ultrasonic nanowelding. RodríguezManzo et al. ${ }^{9)}$ used focused electronic beam to solder cobalt and nickel nano particles with CNTs respectively. Such reactions between nano scale materials are far different from the macro ones because of their size effects and quantum effects, ${ }^{19,20)}$ and the mechanism of a nano-joining process should be understood. In terms of illustrating the mechanisms and depicting the processes of nanojoining, computational method is very powerful. Molecular dynamics (MD) simulation, as one of the most commonly used computational methods, which calculates the trajectories of atoms by solving their Newtonian equations of motion, show great superiority on studying processes in nano scale. MD has long been used to simulate processes related to CNTs and to study their properties. ${ }^{21-24)}$

CNTs with different chiralities show different electrical properties and are thus used in different circumstances. Thus the influence of CNT chirality on its nano-joining process needs to be studied. Metals such as nickel, platinum and gold bond with CNTs not only gently but also efficiently. ${ }^{25,26)}$ In this paper, gold is used as a representative metal to model.

*Corresponding author, E-mail: hitjoining@hit.edu.cn

\section{Simulation Procedure}

Three potentials are used in our model to calculate the force field between atoms. Adaptive intermolecular reactive empirical bond order potential ${ }^{27)}$ is used to calculate the force field between carbon atoms in the CNT and embedded-atom method potential ${ }^{28)}$ is used to calculate the force field between gold atoms. As for the force field between carbon atoms and gold atoms, we choose a novel bond order potential, ${ }^{26}$ ) which has been successful to simulate the deposition of metals on CNTs. ${ }^{29)}$ This potential offers C-M interaction as well as M-M interaction and only the C-M interaction is used in this paper. Even though this potential may not be absolutely accurate, it is adequate for our work which focuses on a comparison issue.

Large-scale Atomic/Molecular Massively Parallel Simulator (LAMMPS) is used as our simulating package. Our box size is $20 \mathrm{~nm} \times 20 \mathrm{~nm} \times 20 \mathrm{~nm}$ and fixed boundary condition is used in each direction. The simulation is run in NVT ensemble, where the initial temperature is $800 \mathrm{~K}$. No pressure is exerted to the system. The diameter of the spherical gold nano particle is $2 \mathrm{~nm}$. Chirality is a special geometrical property of CNTs compared with other nano materials. Properties, especially electrical properties vary a lot from CNT to CNT with different chirality parameters. ${ }^{30)}$ Five $10 \mathrm{~nm}$-long CNTs (diameter all about $1 \mathrm{~nm}$ ) with different chirality parameters of $(5,5),(4,6),(3,7),(1,8)$ and $(0,9)$ are modeled respectively. The initial atom velocities are determined by the Maxwell distribution. Leap-frog algorithm is used to numerically integrate the Newtonian equations of motion with a fixed time step of $1 \mathrm{fs}$.

Center of mass (COM) and mean square displacement (MSD) of gold atoms is used in our model to analyze the deformation and displacement of gold particle respectively. Coordination number of carbon atoms is used to calculate the number and distribution of $\mathrm{C}$-Au bonds.

\section{Results and Discussion}

In a typical bonding process, gold atoms tend to find their equilibrium positions in the middle of hexagons, and jump 


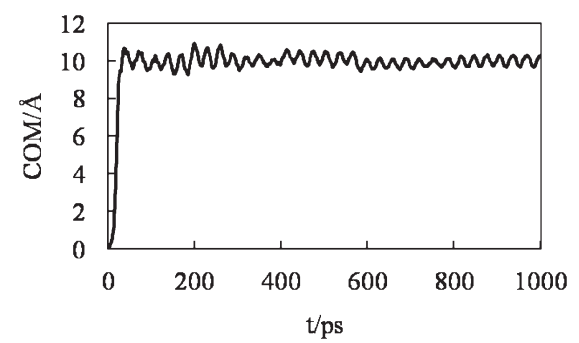

Fig. 1 COM of gold particle in a long time simulation of $1000 \mathrm{ps.}$

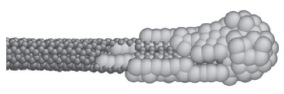

$(5,5)$

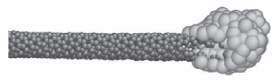

$(1,8)$

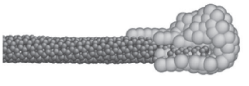

$(4,6)$

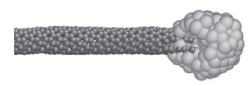

$(0,9)$
Fig. 2 Final screenshots of $(5,5),(4,6),(3,7),(1,8),(0,9)$ CNTs joints with $2 \mathrm{~nm}$ gold particles under $800 \mathrm{~K}$.

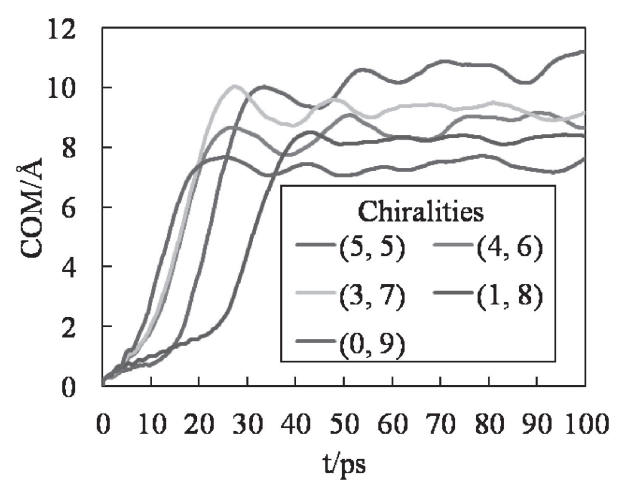

Fig. 3 The COMs of gold particle during the bonding process with different CNTs.

impulsively from one to another. Our previous work shows that the equilibrium length of C-Au bond is $0.203 \mathrm{~nm}^{31)}$ In a long time simulation of $1000 \mathrm{ps}$ in this paper, by calculating the COM of gold nanoparticle (Fig. 1), it can be seen that the system is stable and in the following discussion only the first $100 \mathrm{ps}$ process is focused on.

Due to the large surface curvature of a CNT, its chirality impacts greatly on the arrangement of hexagons. Thus the joints obtained by different chirality CNTs are different, as shown in Fig. 2. By making some atoms invisible in each joint, it can be seen that gold atoms diffuse along not only outer wall but also inner wall of the CNT.

By calculating the COM of gold atoms (Fig. 3), the displacement of a gold particle as a whole can be known. In general, when joining with a typical armchair $(5,5) \mathrm{CNT}$, the gold particle displaces most, about 11 angstroms. With the CNT more like zig-zag type, the gold particle displaces less. When joining with a typical zig-zag $(0,9) \mathrm{CNT}$, the gold particle displaces least, less than 8 angstroms, which is less than with $(5,5)$ CNT by about the diameter of a single gold atom.

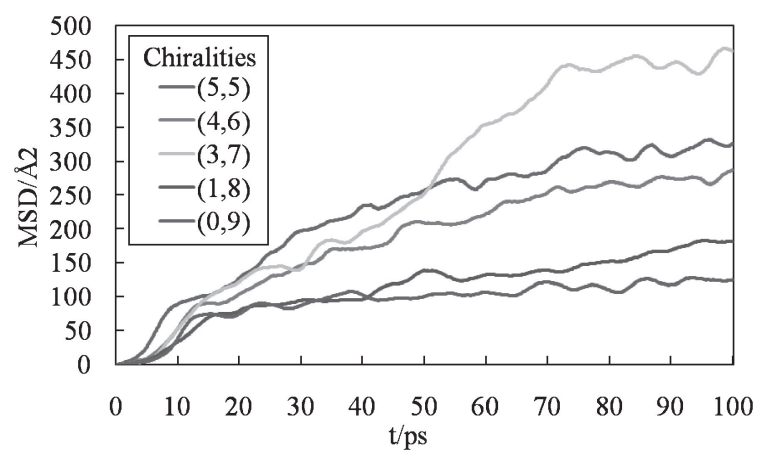

Fig. 4 The MSDs of gold atoms during the bonding process with different CNTs.

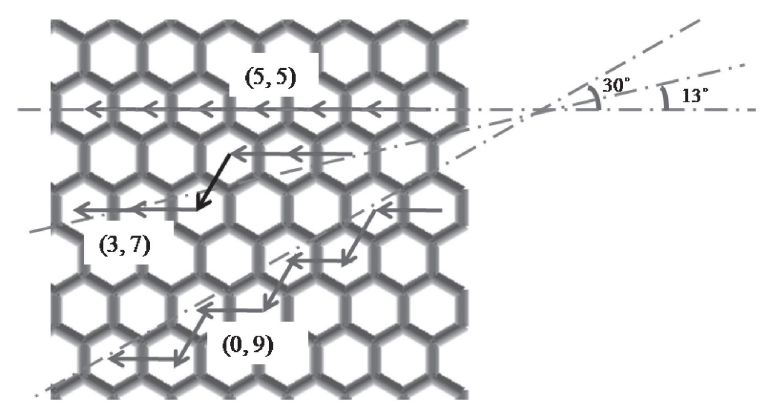

Fig. 5 The diffusing path of a gold atom on three CNTs of $(5,5),(3,7)$ and $(0,9)$, where the arrows indicate the diffusing paths and the green dashdotted lines indicate the CNT axes.

From MSD results (Fig. 4), the extents of deformation of gold particles are different from joint to joint, depending on the chiralities of CNTs. In this aspect, the five CNTs can be classified to three groups. The first one is $(3,7) \mathrm{CNT}$. It has the biggest spirality of all the five specimens, and the gold particle deforms most when joining with it. $(5,5)$ and $(4,6)$ CNTs are armchair and armchair-like CNTs and gold particles deform less when joining with them. $(1,8)$ and $(0,9)$ CNTs are zigzag and zigzag-like CNTs and gold particles deforms least when joining with them.

Typically, the MSD and the COM of gold atoms should be correlated, like the cases of all the CNTs except for $(3,7)$ CNT. However, as can be seen, the $(3,7) \mathrm{CNT}$ is an outlier, whose MSD is the largest rather than $(5,5)$ CNT. This is due to the differences of diffusing paths of gold atoms determined by hexagons arrangement in CNTs, as shown in Fig. 5.

On a typical armchair $(5,5)$ CNT, when a gold atom diffuses from hexagon to hexagon, it goes directly along the axis of the CNT. While a gold atom diffuses on a typical zigzag $(0,9) \mathrm{CNT}$, its path is always 30 degrees from the axis of the CNT from a planar view, which is aggravated on a practical CNT due to its large surface curvature, making it even more difficult for a gold atom to move long along the axis. That is why COM of gold particle is larger with CNT more like armchair type. Regarding MSD, the movements not only along CNT but also in the plane perpendicular to CNT contribute to it and that is the key point making it different from COM. Thus it can be inferred from the above results that $(3,7) \mathrm{CNT}$ makes gold atoms move more than others in the cross-sectional plane. 


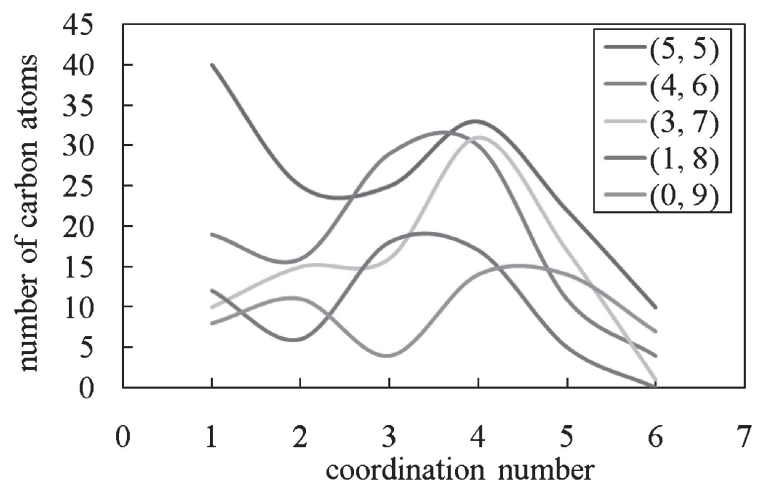

Fig. 6 Coordination number distribution of carbon atoms in each joint.

On a $(3,7)$ CNT, to move along the axis is like moving along a line about 13.0 degree from $(5,5)$ CNT axis. That makes a gold atom move in one circumferential direction instead of move back and forth, as the case of $(0,9)$ CNT. In other word, the movement indicated by the black arrow in Fig. 5 is very difficult to take place. As a result, MSD is increased.

To specify the bonds distribution in the joint, coordination number of carbon atoms is calculated, as shown in Fig. 6. Here the coordination number is defined by the number of gold atoms within 2.2 angstrom of each carbon atom. Generally, total bond number is regularly affected by CNT chirality, where typical armchair $(5,5)$ CNT bonds most with gold atoms and with the CNT chirality closer to zig-zag type, the number of $\mathrm{C}-\mathrm{Au}$ becomes less. And the typical zig-zag $(0,9)$ CNT bonds least with gold atoms. However, the bonds distribution in detail is very much different from joint to joint. Specifically, the main coordination numbers of carbon atoms are 1 and 4 in $(5,5) \mathrm{CNT}, 3$ and 4 in $(4,6) \mathrm{CNT}, 4$ in $(3,7)$ CNT, 3 and 4 in $(1,8) \mathrm{CNT}, 4$ and 5 in $(0,9) \mathrm{CNT}$ respectively. This irregularity is the consequence of both the hexagons distribution of the CNT and the lattice structure of gold atoms. And it is hard to predict which joint is better and which is worse.

\section{Conclusions}

Our MD simulation results show that when bonding with CNTs with different chiralities, the gold nanoparticle behaves differently. When the CNT has high spirality such as a $(3,7)$ CNT, the gold particle deforms more compared with the low spirality CNTs such as armchair and zig-zag ones. The displacement of the particle as a whole is affected by the diffusing path of gold atoms on the CNT, which is determined by the arrangement of hexagons. The bonds distribution shows no regularity from joint to joint, which is the result of the lattice structure of both materials.

To sum up, the CNT chirality influences on the behavior of metal atoms during the joining process as well as the bonds distribution in the final joint. This needs to be taken into consideration in experiments in that not only CNT itself is a big influence on the electrical property of the device, but the joint structure plays an important role as well, which is also affected by the CNT chirality. Thus our results can serve as a important guideline for researchers to do experiments.

\section{Acknowledgements}

The authors gratefully acknowledge the financial support from the National Natural Science Foundation of China (NSFC, Grant Numbers 51105107, 51275135 and 51021002), Natural Science Foundation of Heilongjiang Province (Grant number QC2011C044) and Specialized Research Fund for the Doctoral Program of Higher Education (Grant number 20112302130005).

\section{REFERENCES}

1) S. Iijima and T. Ichihashi: Nature 363 (1993) 603-605.

2) J. Lu and L. Zhang: Comp. Mater. Sci. 35 (2006) 432-441.

3) J. P. Issi, L. Langer, J. Heremans and C. H. Olk: Carbon 33 (1995) 941948.

4) M. S. Ferreira and S. Sanvito: J. Magn. Magn. Mater. 290-291 Part 1 (2005) 286-289.

5) L. Vivien, P. Lançon, D. Riehl, F. Hache and E. Anglaret: Carbon 40 (2002) 1789-1797.

6) Y. Zhou, A. Hu, M. I. Khan, W. Wu, B. Tam and M. Yavuz: J. Phys. Conf. Ser. 165 (2009) 012012.

7) K. Asaka, H. Nakahara and Y. Saito: Appl. Phys. Lett. 92 (2008) 023114.

8) C. Changxin, Y. Lijun, K. Eric Siu-Wai and Z. Yafei: Nanotechnology 17 (2006) 2192-2197.

9) J. A. Rodríguez-Manzo, F. Banhart, M. Terrones, H. Terrones, N. Grobert, P. M. Ajayan, B. G. Sumpter, V. Meunier, M. Wang, Y. Bando and D. Golberg: Proc. National Academy of Sciences 2009 March 24 106 (2009) 4591-4595.

10) H. Tohmyoh: J. Appl. Phys. 105 (2009) 014907.

11) B. Zhao, C. Chen, B. Yadian, P. Liu, Z. Li, D. Xu and Y. Zhang: Thin Solid Films 517 (2009) 2012-2015.

12) M. S. Wang, J. Y. Wang, Q. Chen and L. M. Peng: Adv. Funct. Mater. 15 (2005) 1825-1831.

13) A. P. Graham, G. S. Duesberg, W. Hoenlein, F. Kreupl, M. Liebau, R. Martin, B. Rajasekharan, W. Pamler, R. Seidel, W. Steinhoegl and E. Unger: Appl. Phys. A 80 (2005) 1141-1151.

14) D. Lixin, T. Xinyong, Z. Li, B. J. Nelson and Z. Xiaobin (eds.): Nano/ Micro Engineered and Molecular Systems, 2006 NEMS '06 1st IEEE Int. Conf. on 2006 18-21 Jan. 2006.

15) A. Bachtold, P. Hadley, T. Nakanishi and C. Dekker: Phys. E 16 (2003) $42-46$.

16) F. Banhart: Nano Lett. 1 (2001) 329-332.

17) Z. W. Xu, Q. L. Zhao, T. Sun, L. Q. Guo, R. Wang and S. Dong: J. Mater. Process. Tech. 190 (2007) 397-401.

18) A. M. Cassell, J. A. Raymakers, J. Kong and H. Dai: J. Phys. Chem. B 103 (1999) 6484-6492.

19) C. H. Kiang, M. Endo, P. M. Ajayan, G. Dresselhaus and M. S. Dresselhaus: Phys. Rev. Lett. 81 (1998) 1869-1872.

20) H. J. Choi, J. Ihm, S. G. Louie and M. L. Cohen: Phys. Rev. Lett. 84 (2000) 2917-2920.

21) S. Negi, M. Warrier, S. Chaturvedi and K. Nordlund: Comp. Mater. Sci. 44 (2009) 979-987.

22) Y. Shibuta: Diam. Relat. Mater. 20 (2011) 334-338.

23) L. G. Zhou and S. Q. Shi: Comp. Mater. Sci. 23 (2002) 166-174.

24) M. Joe, M.-W. Moon, J. Oh, K.-H. Lee and K.-R. Lee: Carbon 50 (2012) 404-410.

25) A. N. Andriotis, M. Menon, G. E. Froudakis and J. E. Lowther: Chem. Phys. Lett. 301 (1999) 503-508.

26) Y. Shibuta and S. Maruyama: Comp. Mater. Sci. 39 (2007) 842-848.

27) S. J. Stuart, A. B. Tutein and J. A. Harrison: J. Chem. Phys. 112 (2000) 6472-6486.

28) S. M. Foiles, M. I. Baskes and M. S. Daw: Phys. Rev. B 33 (1986) 7983-7991.

29) S. Inoue and Y. Matsumura: Chem. Phys. Lett. 464 (2008) 160-165.

30) A. Jorio, R. Saito, J. H. Hafner, C. M. Lieber, M. Hunter, T. McClure, G. Dresselhaus and M. S. Dresselhaus: Phys. Rev. Lett. 86 (2001) 1118-1121.

31) P. He, D. Xu, T. Lin and Z. Jiao: Comp. Mater. Sci. 72 (2013) 38-41. 